\title{
Fracture strength and fractographic analysis of zirconia copings treated with four experimental silane primers
}

Type: Article

Abstract:

This study evaluated and compared the effect of new four experimental silane coupling agents on the fracture strength of zirconia copings. The findings were supported with fractographic and finite element analyses. All together 125 zirconia copings with a ?wall thickness of $0.6 \mathrm{~mm}$ were fabricated on identical nickel-chromium master dies and then divided randomly into five groups $(n=25)$. Four test groups were prepared according the experimental silane primer (labeled: OIWA1, OIWA2, OIWA3 and OIWA4) ?and one control group without silanization. The silane monomers used were: ?3-methacryloxypropyltrimethoxysilane (in OIWA1), ?3acryloxypropyltrimethoxysilane (in OIWA2), ?3-?isocyanatopropyltriethoxysilane (in OIWA3) and styrylethyltrimethoxysilane (in OIWA4). Tribochemical sandblasting (silica-coating) treatment was performed to the inner surface of the copings in the ?test groups. All the specimens were silanized at the inner surfaces of the zirconia copings. Self-?adhesive universal resin cement was used to cement the copings to ?the underlying master die. Zirconia copings were vertically loaded on the ?cusp ?area until the first crack failure was occurred using Precision Universal Tester ?at a ?constant crosshead speed of $1 \mathrm{~mm} / \mathrm{min}$. Then, the machine ?was manually controlled to cause more failure ?to further determine the texture of fracture. Three dimensional finite element analysis and fractography were performed to support the fracture strength findings. Based on the finite element analysis results, zirconia silanized with ?3-acryloyloxypropyltrimethoxysilane showed the highest fracture strength with a mean of ?963.75?N (SD 4.5N), while zirconia copings silanized with ?3methacryloyloxypropyltrimethoxysilane showed a mean fracture strength value of ?925.65N (SD 2.4N). Styrylethyltrimethoxysilane-silanised zirconia showed mean fracture strength of $895.95 \mathrm{~N}$ (SD 3.5N). Adding silane coupling agents to the resin-zirconia interface increased the fracture strengths significantly (ANOVA, $p<0.05$ ). Silanization with four new experimental silane primers in vitro produced significantly ?greater fracture strength than the control group not treated with the test silane.?

\begin{tabular}{|c|l|}
\hline Author & $\begin{array}{l}\text { Mustafa, A. A. } \\
\text { Matinlinna, J. P. } \\
\end{array}$ \\
& $\begin{array}{l}\text { Choi, A. H. } \\
\text { Razak, A. A. A. }\end{array}$ \\
\hline Source & Journal of Adhesion Science and Technology \\
\hline ISSN & $0169-4243$ \\
\hline DOI & $10.1080 / 01694243.2012 .701507$ \\
\hline Volume (Issue) & $27(1)$ \\
\hline Page & $68-80$ \\
\hline Year & 2013 \\
\hline
\end{tabular}




\section{Keyword:}

Zirconia, silane coupling agents, tribochemical silica-coating, fracture, strength, fractography, finite element analysis, silica-coated titanium, dental ceramic crown, bond strength, coupling, agents, resin cement, surface modification, oxide ceramics, luting, cement, adhesion, insights

Please Cite As:

URL:

- $\quad$ http://apps.webofknowledge.com search via Accession No >> 000315187700007

- $\quad$ http://www.tandfonline.com/doi/abs/10.1080/01694243.2012.701507\#.UbU 2_k3BZ4 